\title{
Vasculitis primaria del sistema nervioso central, diagnóstico diferencial de demencia subaguda. Caso clínico
}

\author{
Pablo Araya $C^{1}$, Claudio Sacks P², Paula Muñoz V3, \\ Juan Pablo Gigoux $\mathrm{L}^{1}$, Luis $\mathrm{G}$ arcía $\mathrm{F}^{1}$, Gonzalo Matamala $\mathrm{H}^{1}$, \\ Juan Idiáquez $\mathrm{C}^{2}$.

\section{Primary vasculitis of the Central Nervous System. Report of one case}

Primary central nervous system vasculitis is an uncommon and invalidating disease, that has a fatal course if left untreated. We report a 63 year-old woman presenting with a history of two months of cognitive impairment, dysarthria, gait instability and tremor. After four months of evolution a right hemianopsia and a flaccid paresis of upper right limb appeared. A brain biopsy was performed and the histological findings confirmed the suspicion of primary cerebral vasculitis. The patient was treated with cyclophosphamide and prednisone, observing a partial recovery of cognitive and motor function (Rev Méd Chile 2009; 137: 807-10).

(Key words: Cyclophosphamide; Nervous System; Prednisone; Vasculitis, Central Nervous System)

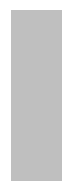

Recibido el 21 de octubre, 2008. Aprobado el 31 de marzo, 2009.

${ }^{1}$ Sección de Neurología, Servicio de Medicina Interna, Hospital Naval Almirante Nef, Viña del Mar, Chile. ${ }^{2}$ Cátedra de Neurología, Escuela de Medicina, Universidad de Valparaíso, Valparaíso, Chile. ${ }^{3}$ Programa de Formación de Especialista en Neurología, Universidad de Valparaíso, Valparaíso, Chile

$\mathrm{L}$ a vasculitis primaria del sistema nervioso central (VPSNC) es una patología de baja frecuencia, potencialmente invalidante y de curso fatal sin tratamiento. Se ha estimado una incidencia de 2,4 por millón de personas ${ }^{1}$, presentándose la mitad de los casos durante la quinta o sexta décadas de la vida. Se diferencia de las vasculitis secundarias por la ausencia clínica y de laboratorio de compromiso sistémico.

$\overline{\text { Correspondencia a: Dra. Paula Muñoz V. Avda. Ricardo }}$ Lyon 1280, Depto. 802, Providencia, Santiago. E mail: pmventurelli@gmail.com
Las manifestaciones son variadas e inespecíficas, siendo más frecuentes la cefalea, alteraciones cognitivas y déficits motores ${ }^{1}$.

Los criterios diagnósticos consideran la presencia de déficit neurológico no explicado por otra causa, asociado a hallazgos angiográficos o histológicos compatibles con vasculitis en el $\mathrm{SNC}^{2}$. Según el patrón angiográfico e histológico se distinguen vasculitis de vaso pequeño, grande $o$ mixtas. Cuando el compromiso es exclusivo de pequeño vaso, la angiografía puede ser normal, lo que dificulta el diagnóstico ${ }^{3}$.

Presentamos a una paciente que desarrolló una demencia subaguda; al realizar el diagnóstico diferencial de una enfermedad de Creutzfeld 
Jacob se confirmó una vasculitis primaria del sistema nervioso central.

\section{Caso Clínico}

Mujer de 63 años, sin antecedentes mórbidos de importancia, que consultó luego de dos meses de evolución de disartria, inestabilidad de la marcha, temblor de extremidades superiores y trastorno del ánimo. Al examen destacó además la presencia de defectos cognitivos (Minimental test score: 21), hiperreflexia difusa, rueda dentada bilateral y marcha a pequeño paso con disminución del braceo. Se le solicitó hemograma, VHS, función renal y hepática, VDRL, estudio de VIH, pruebas tiroideas y anticuerpos antitiroideos, resultando todos normales, salvo una discreta disminución del nivel de vitamina B12 por lo que se inició su reposición. La tomografía computada (TC) de cerebro fue normal.

Algunos días después, se agregaron al cuadro clínico mioclonías multifocales y Babinski bilateral. Por la sospecha de enfermedad de CreutzfeldtJakob (sCJD), se solicitó un electroencefalograma (EEG), donde se apreció una lentitud moderada generalizada, con ritmo delta y theta. La resonancia magnética (RM) de cerebro mostró lesiones bilaterales hiperintensas en sustancia blanca en secuencias T2 y FLAIR, que no se modificaron con administración de gadolinio (Figura 1), y en el líquido cefalorraquideo destacó la presencia de 30 leucocitos por campo, 87\% mononucleares, proteínas $62 \mathrm{mg} / \mathrm{dl}$, glucosa normal y la tinción de Gram y cultivo, ADA y VDRL negativos. Frente a la sospecha de una encefalomielitis diseminada aguda (ADEM), se administraron bolos de metilprednisolona de un gramo diario por cinco días, con lo cual la paciente presentó una leve mejoría.

Sin embargo, tres semanas después la paciente presentó una reagudización progresiva de la sintomatología cognitiva y motora, por lo que se decidió ampliar el estudio: la angiografía por TC cerebral fue normal, se descartaron enfermedades reumatológicas sistémicas: ANA, AntiADN, Ro, La, Sm, RNP, scl70, Jo-1, C3, C4, ANCA (PR3-MPO por ELISA), cuantificación de inmunoglobulinas y electroforesis de proteínas negativos. Anticuerpos antimúsculo liso, antitiroideos, anticardiolipinas y anticoagulante lúpico negativos. El nivel de enzima convertidora de angiotensina fue normal y el estudio de búsqueda de neoplasia oculta resultó negativo.

Dos meses después, la paciente se encontraba postrada, con signología piramidal y extrapiramidal bilateral, mioclonías y verborrea con parafasias. Se le solicitó un nuevo EEG que mostró actividad pseudoperiódica, la que no se evidenció en el EEG de control posterior. El cuadro cedió parcialmente con una nueva dosis de $60 \mathrm{mg}$ de Prednisona diarios por siete días. Durante su evolución la paciente presentó reiteradamente fiebre sin foco infeccioso evidente.

A los cuatro meses de evolución presentó un brusco deterioro con aparición de hemianopsia

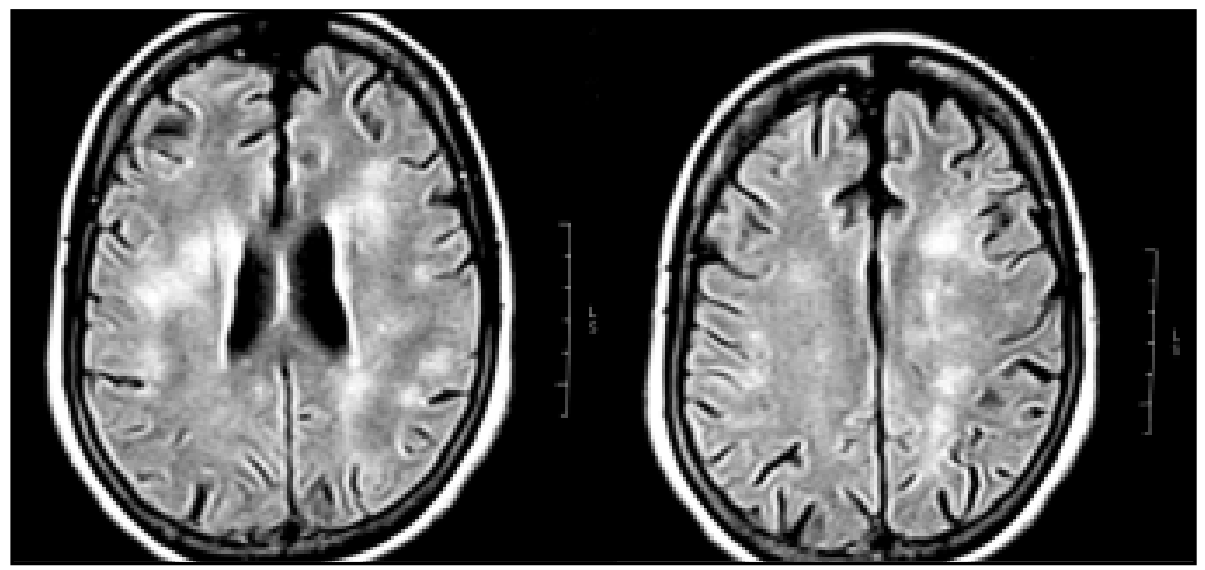

Figura 1. RM de cerebro secuencia FLAIR axial tomada al mes de evolución. Muestra lesiones hiperintensas bilaterales de sustancia blanca en corona radiada y centro semioval. 
homónima derecha y paresia fláccida de la extremidad superior derecha. Se repitió el estudio con RM de cerebro, donde aparecieron hiperintensidades de señal cortical temporooccipital izquierda y múltiples focos subcorticales a nivel de centro semioval y corona radiada, asociados a atrofia cerebral difusa. No presentó captaciones patológicas ni tampoco alteraciones en la difusión ni espectroscopia. El líquido cefalorraquídeo en ese momento presentó 100 leucocitos por campo, de predominio mononuclear, con proteínas de $286 \mathrm{mg} / \mathrm{dl}$ y sin alteración de glucosa. Ante la sospecha de una VPSNC se realizó una biopsia cortical, subcortical y leptomeníngea occipital izquierda, sin angiografía convencional previa. En la histopatología se hizo evidente la presencia de infiltrado mononuclear en la pared de los pequeños vasos parenquimatosos y gliosis reactiva, con preservación de leptomeninges, compatible con una vasculitis cerebral (Figura 2). Se inició tratamiento con ciclofosfamida en pulsos endovenosos de $1 \mathrm{gr} / \mathrm{m}^{2}$ y prednisona oral $60 \mathrm{mg} / \mathrm{dí}$, con lo que la paciente presentó una franca detención del deterioro neurológico con recuperación cognitiva y motora parcial, asociada a disminución de las mioclonías.

\section{DisCusión}

La VPSNC plantea un desafío diagnóstico, más aún cuando se presenta con predominio inicial de pequeño vaso, como en este caso. Esto se refleja en la latencia del diagnóstico, el cual se realiza en promedio 1,2 meses después del inicio de los síntomas, pudiendo demorar 6 meses o más ${ }^{1}$.
El diagnóstico diferencial de un paciente con estas características es amplio. Inicialmente la clínica sugería una sCJD. Otros cuadros con presentación clínica similar son algunas enfermedades neurodegenerativas, como la demencia frontotemporal y la degeneración corticobasal entre otras, que pueden presentar síntomas motores, conductuales, psiquiátricos y cognitivos. También patologías autoinmunes como encefalitis límbica paraneoplásica o no-paraneoplásica, encefalopatía de Hashimoto, esclerosis múltiple, neurosarcoidosis $\mathrm{y}$ algunas infecciones. La RM de cerebro tiene un rol determinante entre los exámenes que permiten diferenciar estos cuadros. Los hallazgos descritos en la RM con técnica de difusión en sCJD probable tienen una sensibilidad de $92 \%$ y especificidad de 94\% para el diagnóstico de esta enfermedad; serían por lo tanto más sensibles que otros exámenes como el EEG, la proteína 14-3-3 en LCR y la Enolasa específica de neuronas ${ }^{4}$. Son excepcionales los casos de sCJD en que se encuentran anomalías en sustancia blanca. Lo expuesto anteriormente hacía menos probable la hipótesis de que la paciente presentada tuviera una SCJD. En un paciente con un cuadro clínico caracterizado por una demencia subaguda, mioclonías e irregularidades al EEG, con estas imágenes en sustancia blanca, se debe descartar una vasculitis de SNC.

El compromiso aislado de vasos pequeños en las VPSNC dificulta el diagnóstico, ya que tanto las manifestaciones clínicas como los estudios no invasivos son muy inespecíficos. Incluso la angiografía es de escasa ayuda: dependiendo de su resolución, puede no evidenciar el compromiso de los vasos más pequeños, requiriéndose de una

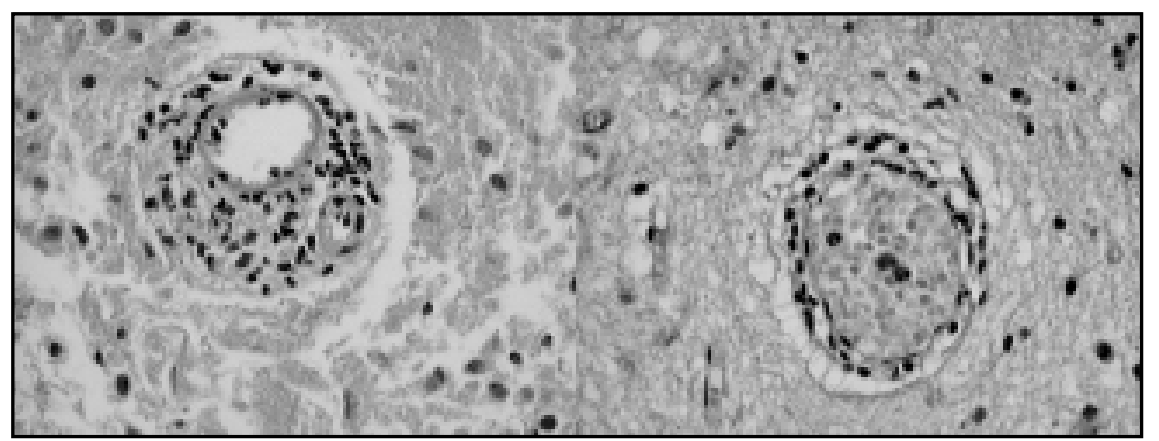

Figura 2. Histopatología de tejido córtico-subcortical occipital izquierdo con tinción de hematoxilina-eosina, 250x. Se observa infiltración de células mononucleares en la pared de los vasos sanguíneos. 
biopsia con histología del tejido afectado para confirmar el diagnóstico ${ }^{3}$. En una reciente revisión retrospectiva de 101 casos de la Clínica Mayo, el diagnóstico fue corroborado por angiografía o por biopsia; de 14 pacientes con diagnóstico confirmado por histología, 8 tuvieron angiografía negativa ${ }^{1}$.

Como el compromiso del SNC puede no ser difuso, se debe considerar también que el estudio patológico puede resultar negativo si la muestra es tomada de una zona indemne. En el caso presentado se contaba con una RM de cerebro donde se evidenciaba compromiso cortical de la región biopsiada.

Los exámenes no invasivos también presentan alteraciones inespecíficas, como la TC de cerebro y la AngioRM. La RM de cerebro estuvo alterada en $97 \%$ de los casos en una serie, en 48 de 87 pacientes mostró infartos cerebrales, generalmente múltiples y bilaterales ${ }^{1}$. Cuando el compromiso de la vasculitis es en vasos pequeños, las lesiones de sustancia blanca en la RM de cerebro son menos específicas, como en el caso presentado, por lo que la sospecha de VPSNC es fundamental para confirmar el diagnóstico.

En cuanto al análisis de líquido cefalorraquídeo, $88 \%$ de los pacientes con VPSNC presentan al menos una alteración citoquímica, predominando la elevación de leucocitos y la proteinorraquia ${ }^{1}$. Una RM cerebral y análisis de LCR normales tendrían un alto valor predictivo negativo para el diagnóstico de $\mathrm{VPSNC}^{5-7}$. La persistente pleocito-

\section{REFERENCIAS}

1. Salvarani C, Brown RD, Calamia KT, Christianson TJ, Weigand SD, Miller DV et al. Primary Central Nervous System Vasculitis: Analysis of 101 Patients. Ann Neurol 2007; 62: 442-51.

2. Calabrese L, Mallek J. Primary angiitis of the central nervous system: report of 8 new cases, review of the literature, and proposal for diagnostic criteria. Medicine (Baltimore) 1988; 67: 20-39.

3. Kadkhodayan Y, Alreshaid A, Morán CJ, Cross DT, Powers WJ, Derdeyn CP. Primary angiitis of the central nervous system at conventional angiography. Radiology 2004; 233: 878-82.

4. Geschwind M, Shu H, Haman A, Sejvar J, Miller B. Rapidly Progressive Dementia. Ann Neurol 2008; 64: 97-108.

5. Calabrese LH, Duna GF. Evaluation and treatment of central nervous system vasculitis. Curr Opin Rheu- sis en el LCR hacía poco probable una SCJD, ya que en esta patología se trata de un hallazgo excepcional $^{8}$.

El pronóstico de la VPSNC ha cambiado drásticamente con el uso de inmunosupresores $^{1,9,10}$. No se han realizado estudios controlados de terapia para establecer cuál es el mejor tratamiento. Están descritos resultados favorables en $81 \%$ de los pacientes tratados inicialmente con corticoides, con la misma respuesta en el grupo al que se le administró ciclofosfamida ${ }^{1}$. La respuesta parcial al tratamiento con corticoides que presentó la paciente constituía otro elemento que alejaba el diagnóstico de SCJD.

No se ha establecido la duración óptima de la terapia en las VPSNC. Con tratamiento se describe una sobrevida de 60\% a 10 años, sin haber una diferencia significativa entre los tratados con prednisona asociada a ciclofosfamida o prednisona sola, y $25 \%$ de recaída ${ }^{1}$. Como en el caso presentado, parece razonable considerar el uso de citotóxicos como la ciclofosfamida en pacientes graves o que no respondan satisfactoriamente a corticoides. Aún existen importantes incógnitas acerca de esta patología, como son su patogenia, criterios diagnósticos específicos y tratamiento.

El caso publicado ejemplifica la dificultad diagnóstica de la VPSNC y la importancia de la sospecha para guiar el estudio y así poder iniciar oportunamente el tratamiento adecuado, evitando las secuelas.

matol 1995; 7: 37-44.

6. Stone JH, Pomper MG, Roubenoff R, Miller TJ, Hellmann DB. Sensitivities of noninvasive tests for central nervous system vasculitis: a comparison of lumbar puncture, computed tomography, and magnetic resonance imaging. J Rheumatol 1994; 21: 1277-82.

7. Calabrese LH, Duna GF, Lie JT. Vasculitis in the central nervous system. Arthritis Rheum 1997; 40: 1189-201.

8. Green A, Sánchez-Juan P, Ladogana A, CuadradoCorrales N, Sánchez-Valle R, Mitrova E et al. CSF analysis in patients with sporadic CJD and other transmissible spongiform encephalopathies. Eur J Neurol 2007; 14: 121-4.

9. Crane R, Kerr LD, Spiera H. Clinical analysis of isolated angiitis of the central nervous system. A report of 11 cases. Arch Intern Med 1991; 151: 2290-4.

10. Moore PM. Diagnosis and management of isolated angiitis of the central nervous system. Neurology 1989; 39: 167-73. 